\title{
MDM2 and CDK4 immunohistochemistry is a valuable tool in the differential diagnosis of low-grade osteosarcomas and other primary fibro-osseous lesions of the bone
}

Fanny Dujardin ${ }^{1}$, Matthieu Bui Nguyen Binh ${ }^{2}$, Corinne Bouvier ${ }^{3}$, Anne Gomez-Brouchet ${ }^{4}$, Frédérique Larousserie ${ }^{2}$, Anne de Muret ${ }^{1}$, Caroline Louis-Brennetot ${ }^{5}$, Alain Aurias ${ }^{5}$, Jean-Michel Coindre ${ }^{6}$, Louis Guillou ${ }^{7}$, Florence Pedeutour ${ }^{8}$, Hélène Duval ${ }^{9}$, Christine Collin ${ }^{10}$ and Gonzague de Pinieux ${ }^{1,2}$

${ }^{1}$ Department of Pathology at Trousseau University Hospital and University François Rabelais, Tours, France;

${ }^{2}$ Department of Pathology, Cochin University Hospital and Paris V René Descartes University, Paris, France;

${ }^{3}$ Department of Pathology, Timone Hospital and Aix Marseille II University, Marseille, France; ${ }^{4}$ Department

of Pathology, Rangueil Hospital, Toulouse, France; ${ }^{5}$ Genetics and Biology of Cancers, Inserm U830, Curie

Institute, Paris, France; ${ }^{6}$ Department of Pathology, Bergonié Institute and Victor Segalen University, Bordeaux,

France; ${ }^{7}$ University Institute of Pathology, Lausanne, Switzerland; ${ }^{8}$ Laboratory of Solid Tumor Genetics, Nice

University Hospital, Nice, France; ${ }^{9}$ Department of Pathology, Pontchaillou University Hospital, Rennes France and ${ }^{10}$ Laboratory of Biochemistry and Molecular Biology, Trousseau University Hospital, Tours, France

Low-grade osteosarcoma is a rare malignancy that may be subdivided into two main subgroups on the basis of location in relation to the bone cortex, that is, parosteal osteosarcoma and low-grade central osteosarcoma. Their histological appearance is quite similar and characterized by spindle cell stroma with low-to-moderate cellularity and well-differentiated anastomosing bone trabeculae. Low-grade osteosarcomas have a simple genetic profile with supernumerary ring chromosomes comprising amplification of chromosome 12q13-15, including the cyclindependent kinase 4 (CDK4) and murine double-minute type 2 (MDM2) gene region. Low-grade osteosarcoma can be confused with fibrous and fibro-osseous lesions such as fibromatosis and fibrous dysplasia on radiological and histological findings. We investigated MDM2-CDK4 immunohistochemical expression in a series of 72 low-grade osteosarcomas and 107 fibrous or fibro-osseous lesions of the bone or paraosseous soft tissue. The MDM2-CDK4 amplification status of low-grade osteosarcoma was also evaluated by comparative genomic hybridization array in 18 cases, and the MDM2 amplification status was evaluated by fluorescence in situ hybridization or quantitative real-time polymerase chain reaction in 31 cases of benign fibrous and fibro-osseous lesions. MDM2-CDK4 immunostaining and MDM2 amplification by fluorescence in situ hybridization or quantitative real-time polymerase chain reaction were investigated in a control group of $\mathbf{2 3}$ cases of primary high-grade bone sarcoma, including 20 conventional high-grade osteosarcomas, two pleomorphic spindle cell sarcomas/malignant fibrous histiocytomas and one leiomyosarcoma. The results showed that MDM2 and/or CDK4 immunoreactivity was present in $89 \%$ of lowgrade osteosarcoma specimens. All benign fibrous and fibro-osseous lesions and the tumors of the control group were negative for MDM2 and CDK4. These results were consistent with the MDM2 and CDK4 amplification results. In conclusion, immunohistochemical expression of MDM2 and CDK4 is specific and provides sensitive markers for the diagnosis of low-grade osteosarcomas, helping to differentiate them from benign fibrous and fibro-osseous lesions, particularly in cases with atypical radio-clinical presentation and/or limited biopsy samples.

Modern Pathology (2011) 24, 624-637; doi:10.1038/modpathol.2010.229; published online 18 February 2011

Keywords: CDK4; immunohistochemistry; low-grade osteosarcoma; MDM2; parosteal osteosarcoma

Correspondence: Dr G de Pinieux, MD, PhD, Department of Pathology, CHU Tours, Service d'Anatomie Pathologique, Hôpital Trousseau, 37044 Tours Cedex 9, France.

E-mail: depinieux@med.univ-tours.fr

Received 29 July 2010; revised 3 November 2010; accepted 3 November 2010; published online 18 February 2011 
Low-grade osteosarcoma is a rare malignancy that represents about $5-7 \%$ of all osteosarcomas and is typically divided into two main subgroups on the basis of location in relation to the bone cortex, that is, parosteal osteosarcoma and low-grade central osteosarcoma. Parosteal osteosarcoma is the most frequent form of low-grade osteosarcoma and represents $4-5 \%$ of all osteosarcomas. It is the most common type of osteosarcoma of the bone surface. It occurs mostly in young adults during the third decade of life, with a slight female predominance. ${ }^{1}$ Most cases (about 70\%) develop on the lower posterior femoral shaft, although some occur on other long bones. ${ }^{2}$ Parosteal osteosarcoma of the flat bones is exceptional. Parosteal osteosarcoma is a slow growing tumor revealed by the appearance of a palpable mass, pain or swelling. Radiographic studies show a heavily mineralized mass attached to the cortex with a broad base, and the tumor has a tendency to wrap around the involved bone. ${ }^{1}$ Lowgrade central osteosarcoma accounts for $1-2 \%$ of all osteosarcomas. Men and women are equally affected and the peak incidence occurs in the second and third decades of life. Approximately $80 \%$ of these tumors are located in the long bones, with a distinct predilection for the distal femur and proximal tibia. ${ }^{1,3}$ The radiographic appearance of low-grade central osteosarcoma is highly variable at presentation, but the majority of these tumors will show some degree of cortical disruption, with or without extension to soft tissue. ${ }^{1}$ Nevertheless, a significant number of these lesions may initially present a misleading radiographic appearance, suggestive of a benign lesion. Parosteal osteosarcoma and low-grade central osteosarcoma share the same histological appearance. ${ }^{4}$ They are characterized by a spindle cell stroma with low-to-moderate cellularity and fairly mature bone trabeculae. The stromal cells resemble fibroblasts or myofibroblasts, are minimally cytologically atypical and have low mitotic activity. Areas composed of stroma can be prominent, with rare ossification clusters, and may mimic fibromatosis or a desmoplastic fibroma. More than $50 \%$ of parosteal osteosarcomas have a significant cartilaginous tumor component. ${ }^{1}$ Eighteen percent of low-grade central osteosarcomas also have cartilage differentiation, seen as small scattered foci of atypical cartilage. ${ }^{3}$ The clinical behavior of both parosteal osteosarcoma and low-grade central osteosarcoma is favorable and indistinguishable, with identical rates of local recurrence $(7 \%)$ and 5 -year survival above $80 \% .^{2,5,6}$ The prognosis is mainly conditional on the risk of local recurrence after inadequate resection and dedifferentiation. ${ }^{6-9}$ Unless the tumor is dedifferentiated, it does not metastasize. ${ }^{2}$ About 15-29\% of low-grade osteosarcomas will show dedifferentiation. ${ }^{1,3,7,10}$ Dedifferentiated low-grade osteosarcomas are defined by the presence of areas of undifferentiated spindle cells and/or pleomorphic sarcoma (ie, fibrosarcoma or malignant fibrous histiocytoma) or high-grade osteosarcoma. ${ }^{3,7,10,11}$ Wide surgical excision is recommended for primary lesions, and adjuvant chemotherapy is reserved for dedifferentiated lesions. ${ }^{5}$

Several studies have documented difficulties in the diagnosis of low-grade osteosarcoma. ${ }^{2,3,8,9,12}$ Inability to diagnose the lesion correctly often leads to inadequate initial operative procedures. In two reviews of the literature, low-grade osteosarcomas were initially misdiagnosed as benign tumors in $32-60 \%$ of cases. ${ }^{13,14}$ In the Mankin series, the rate of misdiagnosis reached $50 \%$ in specialist centers. ${ }^{14}$ The differential diagnosis may include diverse entities such as fibrous dysplasia, non-ossifying fibroma, myositis ossificans, fracture callus, ossifying hematoma, osteochondroma, desmoplastic fibroma, osteoma and giant bone island. ${ }^{2-4,6,12,15}$ Little information on low-grade osteosarcoma genetics is available in the literature. These tumors seem to share similarities in changes in cytogenetics and molecular genetics involving ring or giant marker chromosomes, which contain amplification of the 12q13-15 region, including murine doubleminute type 2 (MDM2) and cyclin-dependent kinase 4 (CDK4). ${ }^{16-21}$

The purpose of this study was to determine the value of MDM2 and CDK4 immunostaining for the differential diagnosis of low-grade osteosarcomas and benign fibrous or fibro-osseous lesions. We then investigated the immunohistochemical status of 72 low-grade osteosarcomas and 107 fibrous or fibro-osseous lesions of the bone or paraosseous soft tissue. We also analyzed MDM2 and CDK4 amplification levels in 18 cases of low-grade osteosarcoma by comparative genomic hybridization array (aCGH) and MDM2 amplification of 31 fibrous/ fibro-osseous lesions by real-time quantitative polymerase chain reaction (Q-PCR) $(n=16)$ or fluorescence in situ hybridization (FISH) $(n=15)$, depending on the material available. A control group of 23 cases of primary high-grade bone sarcoma, including 20 conventional high-grade osteosarcomas, two pleomorphic spindle cell sarcomas/malignant fibrous histiocytomas and one leiomyosarcoma, were evaluated for both MDM2 and CDK4 by immunohistochemistry and for MDM2 by molecular study (FISH or Q-PCR).

\section{Materials and methods}

\section{Tumor Specimens}

Institutional ethical guidelines were followed for this retrospective study. All cases (including lowgrade osteosarcomas, fibrous and fibro-osseous bone lesions and the control group of high-grade sarcomas) came from the database of the French Bone Pathology Group (Cochin Hospital, Paris; Trousseau Hospital, Tours; Timone Hospital, Marseille; Rangueil Hospital, Toulouse; Bergonié Institute, Bordeaux; Pontchaillou Hospital, Rennes) and from the University Institute of Pathology, Lausanne, 
Switzerland. They were reviewed by three pathologists (MBNB, FD and GdP). Formalin-fixed and paraffin-embedded tissues were available from all tumors, and snap-frozen material (stored at $-80^{\circ} \mathrm{C}$ ) was available in 32 cases (18 low-grade osteosarcomas, 11 fibro-osseous lesions and three high-grade sarcomas). Bony specimens were decalcified according to a standardized protocol with $6.5 \%$ nitric acid or rapid bone decalcifier (hydrochloric acid-based product) rotating with a longer period of fixation in buffered formalin. We finally collected 72 cases (31 male, 41 female) of low-grade osteosarcoma/dedifferentiated low-grade osteosarcoma divided into 54 primary low-grade osteosarcomas and 18 dedifferentiated low-grade osteosarcomas, including 64 parosteal osteosarcomas and eight low-grade central osteosarcomas. All samples originated from biopsy, except for six samples of low-grade osteosarcoma/ dedifferentiated low-grade osteosarcoma, which originated from surgical resection specimens. Mean age at presentation was 33.1 years (range 10-78 years). Long bones were the site of involvement for 64 patients, and the knee area (distal femur, 45 cases and proximal tibia, four cases) was the main site. The clinical symptoms were nonspecific and the diagnosis was confirmed by histological analysis correlated with radiographic features in most cases. The initial histological diagnosis in two cases with atypical radio-clinical presentation included desmoplastic fibroma and osteofibrous dysplasia. One case was initially misdiagnosed as myositis ossificans. The histological features of low-grade osteosarcoma included well-delineated long bone trabeculae separated by a spindled fibrous stroma (Figure 1). The stroma had to be devoid of cytological abnormalities to be considered low grade. Ten low-grade osteosarcomas had a particular growth pattern, seven lesions simulating the appearance of fibrous dysplasia (irregular bone trabeculae

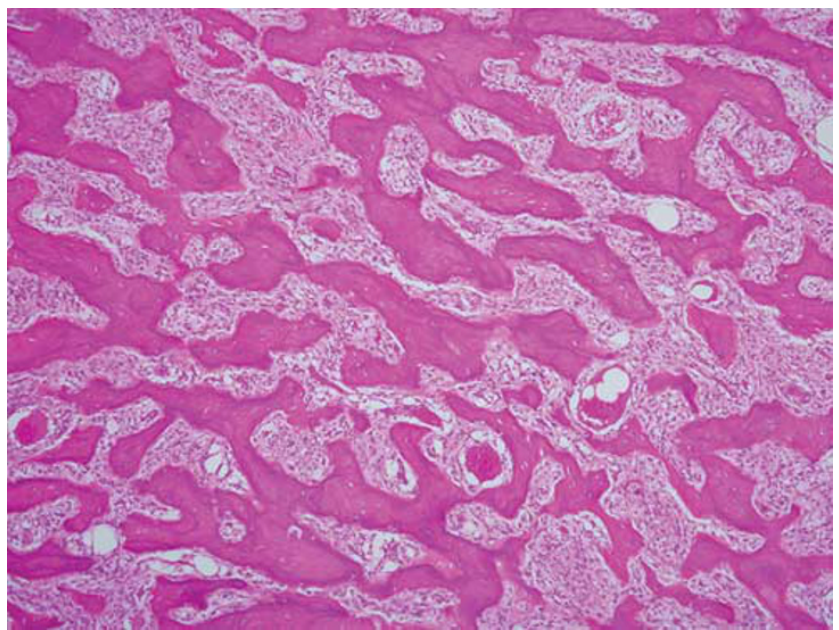

Figure 1 Low power magnification showing the typical, well-differentiated osseous trabeculae and spindle cell stroma seen in low-grade osteosarcoma (hematoxylin and eosin staining, original magnification, hematoxylin-eosin-safran $($ HES $) \times 40$ ). resembling 'Chinese characters' in a spindle cell stroma) (Figure 2), two presenting as desmoid-type fibromatosis (prominent spindle cell proliferation with heavy collagenization and only scattered bone trabeculae) (Figure 3) and one as osteofibrous dysplasia. In 18 patients, there were areas of dedifferentiation at presentation (synchronous, $n=17$ ) (Figure $4 \mathrm{a}$ ) or at the time of recurrence (metachronous, $n=1$ ). These dedifferentiated low-grade osteosarcomas included 12 high-grade osteosarcomas and four malignant fibrous histiocytomas (Figure $4 \mathrm{~b}$ ), and two were unspecified. The majority of dedifferentiated low-grade osteosarcomas dedifferentiated into high-grade osteosarcomas that were osteoblastic subtype, but two cases were classified as chondroblastic subtype.

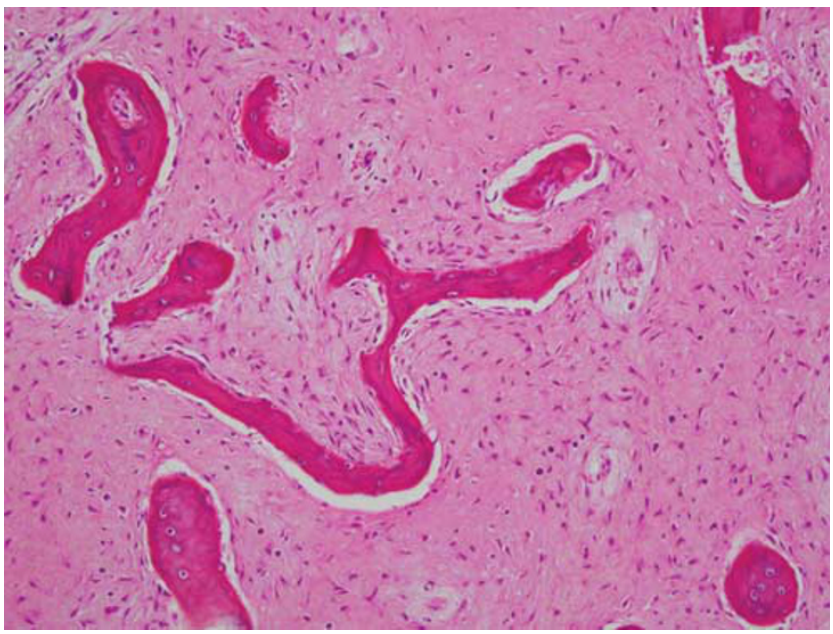

Figure 2 Fibrous dysplasia-like area in a low-grade osteosarcoma with characteristic irregularly-shaped bony trabeculae (hematoxylin-eosin-safran (HES) $\times 200$ ).

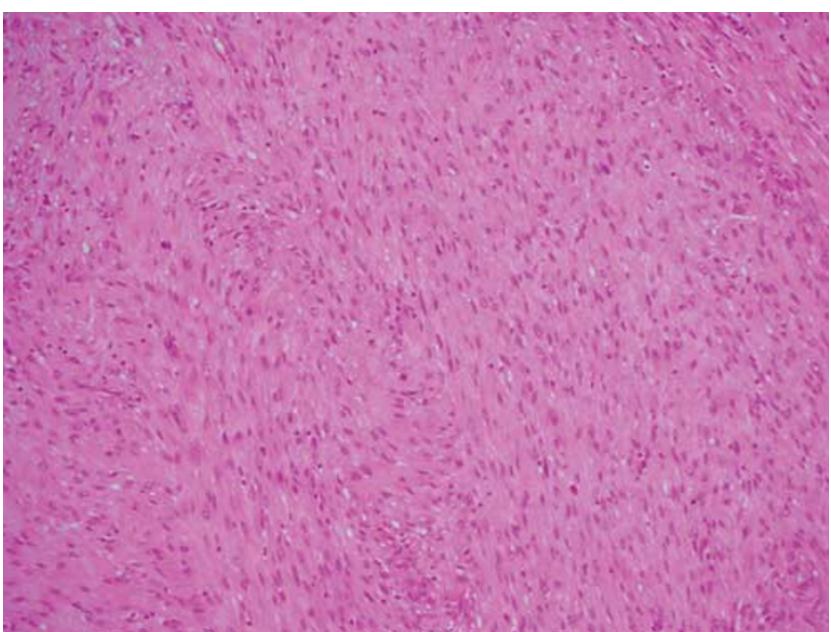

Figure 3 Desmoid-like area in a low-grade central osteosarcoma: microscopically, it was a neoplasm composed of a fibrous stroma containing fibroblast-like cells showing no pleomorphism (hematoxylin-eosin-safran $(\mathrm{HES}) \times 100$ ). 

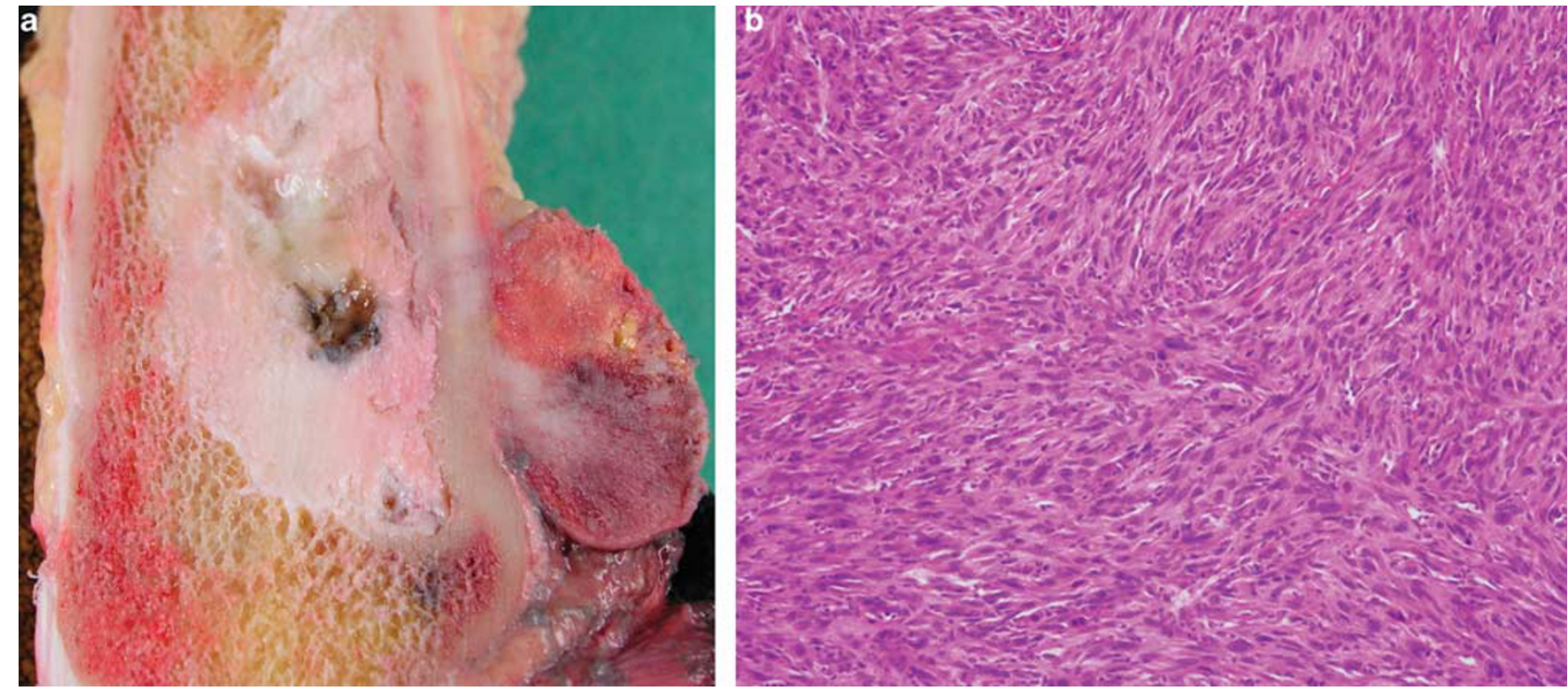

Figure 4 Dedifferentiated parosteal osteosarcoma: a gross specimen viewed coronally (a) shows an ossified, hard bone tumor attached to the posterior cortex of the femur. Intramedullary invasion and dedifferentiation areas with a white fibrous tumor component can be seen. High-grade pleiomorphic spindle cell sarcomatous/malignant fibrous histiocytoma dedifferentiated areas were observed (b) (hematoxylin-eosin-safran (HES) × 200).

Table 1 MDM2 and CDK4 expression on immunohistochemistry in 107 benign fibrous and fibro-osseous bone lesions

\begin{tabular}{|c|c|c|c|}
\hline Histological diagnosis & $\begin{array}{c}\text { Total } \\
\text { number } \\
\text { of } \\
\text { tumors }\end{array}$ & $\begin{array}{c}\text { MDM2- } \\
\text { positive } \\
\text { cases }\end{array}$ & $\begin{array}{c}\text { CDK4- } \\
\text { positive } \\
\text { cases }\end{array}$ \\
\hline Fibrous dysplasia & 44 & 0 & $\begin{array}{c}0 \\
1 \text { non- } \\
\text { inter- } \\
\text { pretable }\end{array}$ \\
\hline Liposclerosing myxofibrous tumor & 4 & 0 & 0 \\
\hline Non-ossifying fibroma & 10 & 0 & 0 \\
\hline $\begin{array}{l}\text { Myositis ossificans and related } \\
\text { lesions }\end{array}$ & 7 & 0 & 0 \\
\hline Callus & 3 & 0 & 0 \\
\hline $\begin{array}{l}\text { Fibro-osseous reparative lesion of } \\
\text { the rib }\end{array}$ & 3 & 0 & 0 \\
\hline Desmoplastic fibroma & 4 & 0 & 0 \\
\hline Aneurysmal bone cyst (solid areas) & 3 & 0 & 0 \\
\hline $\begin{array}{l}\text { Fibrous and fibro-osseous lesions } \\
\text { of jaws }\end{array}$ & 10 & 0 & 0 \\
\hline Nora's lesion & 6 & 0 & 0 \\
\hline Osteofibrous dysplasia & 4 & 0 & 0 \\
\hline Chronic osteitis & 2 & 0 & 0 \\
\hline $\begin{array}{l}\text { Ossifications (metaplastic or } \\
\text { reactive) }\end{array}$ & 2 & 0 & 0 \\
\hline $\begin{array}{l}\text { Adamantinoma osteofibrous } \\
\text { dysplasia-like }\end{array}$ & 1 & 0 & 0 \\
\hline Paget's disease & 3 & 0 & 0 \\
\hline Desmoid tumor & 1 & 0 & 0 \\
\hline
\end{tabular}

Abbreviations: CDK4, cyclin-dependent kinase 4; MDM2, murine double-minute type 2 .

In addition, we included 107 benign fibrous or fibro-osseous lesions of the bone or paraosseous soft tissue (Table 1): 44 cases of fibrous dysplasia, 11 with secondary aneurysmal bone cysts, two with cartilaginous differentiation and two with psammomatoid variants, four cases of liposclerosing myxofibrous tumor, one osteofibrous dysplasia-like adamantinoma, four desmoplastic fibromas, 10 nonossifying fibromas, three solid variants of aneurysmal bone cysts, six Nora's lesions, four osteofibrous dysplasia, three fracture callus, two chronic osteitis, three fibro-osseous reparative lesions of the rib, three Paget's disease, one ectopic metaplastic bony tissue, one reactive periosteal osteogenesis, one desmoid-type fibromatosis of soft tissue with bone destruction, 10 maxillo-facial fibro-osseous lesions (six ossifying fibroma, two fibrous dysplasia, one osseous dysplasia and one solitary myofibroma), six myositis ossificans and one florid reactive periostitis. Furthermore, 20 conventional primary high-grade osteosarcomas, two pleomorphic spindle cell sarcomas/malignant fibrous histiocytomas of the bone and one intra-osseous leiomyosarcoma were investigated as a control group.

\section{Immunohistochemistry}

For MDM2 and CDK4 immunostaining, 4- $\mu$ m-thick paraffin sections were cut and mounted on glass slides (Superfrost $+{ }^{\mathbb{B}}$, Menzel Glazer). Preparations were dried for $1 \mathrm{~h}$ at $58^{\circ} \mathrm{C}$, then overnight at $37^{\circ} \mathrm{C}$, and sections were then deparaffinized with toluene and rehydrated with ethanol. Preparations were pretreated with EDTA (MS-Unmasker-Diapath, Microstain division, Martinengo, Italy), and a heatbased antigen retrieval method was used before incubation. Endogenous peroxidase was blocked using $3 \% \mathrm{H}_{2} \mathrm{O}_{2}$ solution for $5 \mathrm{~min}$. The primary antibodies used were: MDM2 (Zymed Laboratories, South San Francisco, CA, USA; clone IF2) dilution 
1:25, and CDK4 (Biosource International, Camarillo, CA, USA; clone DCS-31) dilution 1:50. Sections were incubated with the primary antibodies for $1 \mathrm{~h}$ at $22^{\circ} \mathrm{C}$, followed by staining with the Envision kit (Dako, Carpinteria, CA, USA). Sections were then revealed in a diaminobenzidine solution for $7 \mathrm{~min}$ and stained with hematoxylin for $16 \mathrm{~s}$. This analysis was used for the three subgroups (low-grade osteosarcomas, fibrous and fibro-osseous lesions and control group of high-grade bone sarcomas). For dedifferentiated low-grade osteosarcomas, the samples were obtained from well-differentiated and dedifferentiated components. Immunostaining was performed on whole-tissue sections and slides were evaluated by three independent pathologists (MBNB, FD and GdP). Discordant cases were reevaluated collegially. The stained MDM2-CDK4 slides were viewed at low magnification to identify areas potentially containing the greatest number of positive cells. Ten high power fields $(\times 400$ magnification) were then counted in these areas. A tumor was considered as MDM2 or CDK4 positive when at least one cell nucleus was stained per high power field. The percentage of positive tumor cell nuclei was evaluated approximately by visual scanning of the slides at medium power. Four classes of immunostaining positivity were defined: $\leq 10$, $11-25,26-50$ and $>50 \%$. The negative control was provided by the molecular results (CGH, Q-PCR and FISH analysis) when material was available and the technology feasible.

\section{Molecular Analysis}

\section{CGH analysis}

Eighteen low-grade osteosarcomas for which frozen tissue from the tumor was available were studied using aCGH developed at INSERM U830, Institut Curie, Paris, France. DNA was extracted from frozen samples using a standard phenol-chloroform procedure. After digestion with DpnII (Ozyme, Saint Quentin en Yvelines, France) and column purification (Qiaquick PCR purification kit; Qiagen, Courtaboeuf, France), tumor DNA for each sample was labeled by the random priming method (Bioprime DNA labeling system; Invitrogen, Cergy-Pontoise, France) incorporating dCTP-cyanine-5 (PerkinElmer, Wellesley, MA, USA). Using the same procedure, we labeled control DNA incorporating dCTPcyanine-3 (Perkin-Elmer, Wellesley, MA, USA). After ethanol co-precipitation with $210 \mu \mathrm{g}$ of Human Cot-1 DNA (Invitrogen, Cergy-Pontoise, France), resuspension in hybridization buffer $(50 \%$ formamide), denaturation at $95^{\circ} \mathrm{C}$ for $10 \mathrm{~min}$ and prehybridization at $37^{\circ} \mathrm{C}$ for $90 \mathrm{~min}$, probes were cohybridized on aCGH. The aCGH slide was previously preblocked with a buffer containing $2.6 \mathrm{mg}$ succinic anhydride/118 ml $\mathrm{N}$-methyl-2-pyrrolidinone/32 ml sodium tetraborate decahydrate, $\mathrm{pH}$ 8.0 (SigmaAldrich, Lyon, France). The aCGH developed in the laboratory contains 3342 sequence-validated BACs. After washing, arrays were scanned using a Genepix 4000B scanner (Axon, Union City, CA, USA). Image analysis was performed with the Genepix 5.1 software (Axon) and ratios of Cy5/Cy3 signals were determined after normalization with the MANOR algorithm (Neuvial2006 BMC bioinformatics).

\section{FISH analysis}

FISH was performed on 15 benign fibro-osseous lesions (11 cases of fibrous dysplasia, one desmoplastic fibroma, two osteofibrous dysplasia and one florid reactive periostitis) and 20 cases of conventional high-grade osteosarcoma. Five- $\mu \mathrm{m}$-thick sections of the formalin-fixed, paraffin-embedded decalcified tissues on silanized slides were deparaffinized for $3 \times 10 \mathrm{~min}$ in xylene, washed in $100 \%$ ethanol, air-dried, incubated in $2 \times$ SSC (sodium saline citrate) at $72^{\circ} \mathrm{C}$ for $40 \mathrm{~min}$, incubated in a proteinase K solution $(500 \mu \mathrm{g} / \mathrm{ml}$ in $2 \times$ SSC; Roche, Meylan, France) at $45^{\circ} \mathrm{C}$ for $5-80 \mathrm{~min}$, washed in $2 \times$ SSC for $2 \times 3 \mathrm{~min}$ at room temperature and stored in $70 \%$ ethanol at $4{ }^{\circ} \mathrm{C}$. For each slide, $200 \mathrm{ng}$ of the biotin-labeled BAC clone RP11-775J10 containing the MDM2 gene was hybridized according to standard procedures. A minimum of 100 nuclei were visualized per slide. The number of fluorescent signals was evaluated for each nucleus analyzed. If at least one or two bright fluorescent spots per nucleus could not be seen on at least $80 \%$ of cells, the result was considered to be uninterpretable (ie, failure of hybridization owing to inappropriate deproteinization conditions). Amplification was defined as more than five fluorescent signals per cell. For each series of experiments, a control slide from a case of well-differentiated liposarcoma known to be positive for MDM2 amplification was also examined.

\section{$Q-P C R$}

Q-PCR was performed on 16 other benign fibroosseous lesions (10 cases of fibrous dysplasia, one desmoid-type fibromatosis, one non-ossifying fibroma, one Nora's lesion, one fracture callus, one solitary myofibroma and one myositis ossificans) and three high-grade bone sarcomas (control group). The presence of a tumor on the frozen specimens used for Q-PCR was investigated by obtaining an HES-stained frozen section. DNA was prepared from frozen tissue samples using the QiAmp DNA mini Kit from Qiagen. Q-PCR was performed using a LightCycler 480 (Roche) to analyze the amplification status of MDM2. Genomic amplification was performed with a starting amount of DNA ( $50 \mathrm{ng}$ ) using the LightCycler 480 Sybr Green I Master kit (Roche). Primer sequences for albumin (used as reference gene) and MDM2 were: MDM2 forward, 5'-CCGGAT GATCGCAGGTG-3'; MDM2 reverse, 5'-AAAAGCTG AGTCAACCTGCCC-3'; Albumin forward, 5'-TGAA ACATACGTTCCCAAAGAGTTT-3'; Albumin reverse, 
5'-CTCTCCTTCTCAGAAAGTGTGCATAT-3'. PCR was carried out as follows: after an initial $5 \mathrm{~min}$ preincubation step at $95^{\circ} \mathrm{C}, 45$ amplification cycles were run, each consisting of $10 \mathrm{~s}$ at $95^{\circ} \mathrm{C}, 10 \mathrm{~s}$ at $60^{\circ} \mathrm{C}$ and $10 \mathrm{~s}$ at $72{ }^{\circ} \mathrm{C}$. The relative amount of gene was compared with the reference gene (Albumin) and calculated with LightCycler Relative Quantification software (Roche).

\section{Statistical Analysis}

The specificity and sensitivity for MDM2 and CDK4 immunostaining were evaluated in low-grade osteosarcomas with or without the dedifferentiation subgroup and compared with that of the fibrous and fibro-osseous lesions subgroup. We hypothesized that low-grade osteosarcoma with or without dedifferentiation would show amplification or immunostaining different from that of other bone lesions studied. MDM2 and CDK4 sensitivity was calculated as the ratio of positively immunostained low-grade osteosarcomas to the total number of lowgrade osteosarcomas examined. MDM2 and CDK4 specificity was calculated as the ratio of unstained non-low-grade osteosarcoma lesions to the total number of non-low-grade osteosarcoma lesions.

The Youden index is the sum of sensitivity and specificity -1.0. It expresses the overall power of the test, the best index being the closest to 1.0. We could not study the correlation between MDM2 amplification detected by FISH, CGH or Q-PCR analysis and MDM2-CDK4 immunostaining because of the non-matching of the series.

\section{Results}

\section{Immunohistochemistry Analysis}

Histological slides from 72 patients with low-grade osteosarcoma/dedifferentiated low-grade osteosarcoma were reviewed. MDM2 and CDK4 staining is summarized in Table 2 according to histology. Only nuclear staining was considered to be a positive result. Immunohistochemistry analysis was interpretable in all low-grade osteosarcomas. MDM2 and/ or CDK4 were expressed in $89 \%$ of low-grade osteosarcomas (64/72) (sensitivity). Each antibody stained $82 \%$ of cases (59/72) and $75 \%$ of low-grade osteosarcomas were stained with both antibodies. Nine of ten low-grade osteosarcomas with a particular growth pattern simulating benign fibrous or fibro-osseous lesions showed MDM2 and/or CDK4 immunostaining. The percentages of positive tumor cell nuclei, according to the four classes of immunostaining positivity $(\leq 10,11-25,26-50$, $>50 \%$ ), are set out separately for MDM2 and CDK4 and for low-grade osteosarcomas and dedifferentiated low-grade osteosarcomas in Tables 3 and 4 , respectively. The percentage of tumor cell MDM2-stained nuclei did not exceed $10 \%$ in any

Table 3 Results of immunohistochemical positivity of MDM2 and $C D K 4$ in low-grade osteosarcomas/dedifferentiated low-grade osteosarcomas $(n=72)$

\begin{tabular}{lcc}
\hline Percentage of nuclear staining & MDM2 & CDK4 \\
\hline$\leq 10$ & $59(100 \%)$ & $17(29 \%)$ \\
$11-25$ & 0 & $42(71 \%)$ \\
$26-50$ & 0 & 0 \\
$>50$ & 0 & 0 \\
Total (positive low-grade & $59 / 72(82 \%)$ & $59 / 72(82 \%)$ \\
osteosarcomas) & &
\end{tabular}

Abbreviations: CDK4, cyclin-dependent kinase 4; MDM2, murine double-minute type 2 .

Table 4 Results of immunohistochemical positivity of MDM2 and $C D K 4$ in dedifferentiated areas of dedifferentiated low-grade osteosarcomas $(n=18)$

\begin{tabular}{lcc}
\hline Percentage of nuclear staining & MDM2 & CDK4 \\
\hline$\leq 10$ & $6(46 \%)$ & $1(6 \%)$ \\
$11-25$ & $7(54 \%)$ & $3(19 \%)$ \\
$26-50$ & 0 & $8(50 \%)$ \\
$>50$ & 0 & $4(25 \%)$ \\
Total (positive dedifferentiated & $13 / 18(72 \%)$ & $16 / 18(88 \%)$ \\
low-grade osteosarcomas) & &
\end{tabular}

Abbreviations: CDK4, cyclin-dependent kinase 4; MDM2, murine double-minute type 2 .

Table 2 MDM2 and CDK4 expression on immunohistochemistry in parosteal and low-grade central osteosarcomas (low-grade osteosarcomas) and dedifferentiated low-grade osteosarcomas

\begin{tabular}{|c|c|c|c|c|c|}
\hline Histological diagnosis & $\begin{array}{l}\text { Total } \\
\text { number of } \\
\text { tumors }\end{array}$ & $\begin{array}{l}\text { MDM2- } \\
\text { positive } \\
\text { cases }\end{array}$ & $\begin{array}{l}\text { CDK4- } \\
\text { positive } \\
\text { cases }\end{array}$ & $\begin{array}{l}\text { MDM2- and } \\
\text { CDK4-positive } \\
\text { cases }\end{array}$ & $\begin{array}{c}\text { MDM2- and/or } \\
\text { CDK4-positive } \\
\text { cases }\end{array}$ \\
\hline Low-grade parosteal osteosarcoma & 48 & $40(83 \%)$ & $37(77 \%)$ & $35(73 \%)$ & $42(87 \%)$ \\
\hline Low-grade central osteosarcoma & 6 & $6(100 \%)$ & $6(100 \%)$ & $6(100 \%)$ & $6(100 \%)$ \\
\hline $\begin{array}{l}\text { Dedifferentiated parosteal and low-grade central } \\
\text { osteosarcomas }\end{array}$ & 18 & $13(72 \%)$ & $16(88 \%)$ & $13(72 \%)$ & $16(88 \%)$ \\
\hline \multicolumn{6}{|l|}{ Total } \\
\hline $\begin{array}{l}\text { Low-grade osteosarcoma/dedifferentiated low-grade } \\
\text { osteosarcoma }\end{array}$ & 72 & $59(82 \%)$ & $59(82 \%)$ & $54(75 \%)$ & $64(89 \%)$ \\
\hline
\end{tabular}

Abbreviations: CDK4, cyclin-dependent kinase 4; MDM2, murine double-minute type 2. 
well-differentiated area of low-grade osteosarcomas (Figure 5a) and they were relatively evenly distributed across any given lesion (Table 3), whereas the proportion of MDM2-stained nuclei was higher in dedifferentiated areas (between 11 and 25\% for more than $50 \%$ of cases) (Table 4). There were more CDK4- than MDM2-stained nuclei in most cases (between 11 and $25 \%$ for $71 \%$ of low-grade osteosarcomas studied) (Figure 5b) (Table 3). CDK4 staining was also more diffuse in dedifferentiated low-grade osteosarcomas, with more than $25 \%$ of stained nuclei in $75 \%$ of cases (Table 4). Finally, the percentages of both MDM2- (Figure 6a) and CDK4- (Figure 6b) stained nuclei were higher in dedifferentiated areas.

Negative immunohistochemistry staining was observed in all cases $(100 \%)$ in the subgroup of benign fibrous and fibro-osseous bone or soft-tissue lesions (specificity). Only one result was not interpretable for CDK4 (fibrous dysplasia). MDM2 and CDK4 were not expressed in the control group (primary highgrade osteosarcomas and high-grade bone sarcomas). MDM2 and CDK4 specificity was $100 \%$ in low-grade osteosarcomas. The Youden index (0.89 for MDM2 and/or CDK4 expression) confirmed the power of the test.

\section{CGH Analysis}

CGH analysis was successfully performed in 18 cases of low-grade osteosarcoma, for which frozen tissues were available. All cases showed an amplification of chromosome 12q13-15 as recurrent genomic imbalance. BACs containing MDM2 and CDK4 genes were amplified (Figure 7). All samples showed amplification of MDM2 and CDK4, whereas $2 / 18$ of these low-grade osteosarcomas
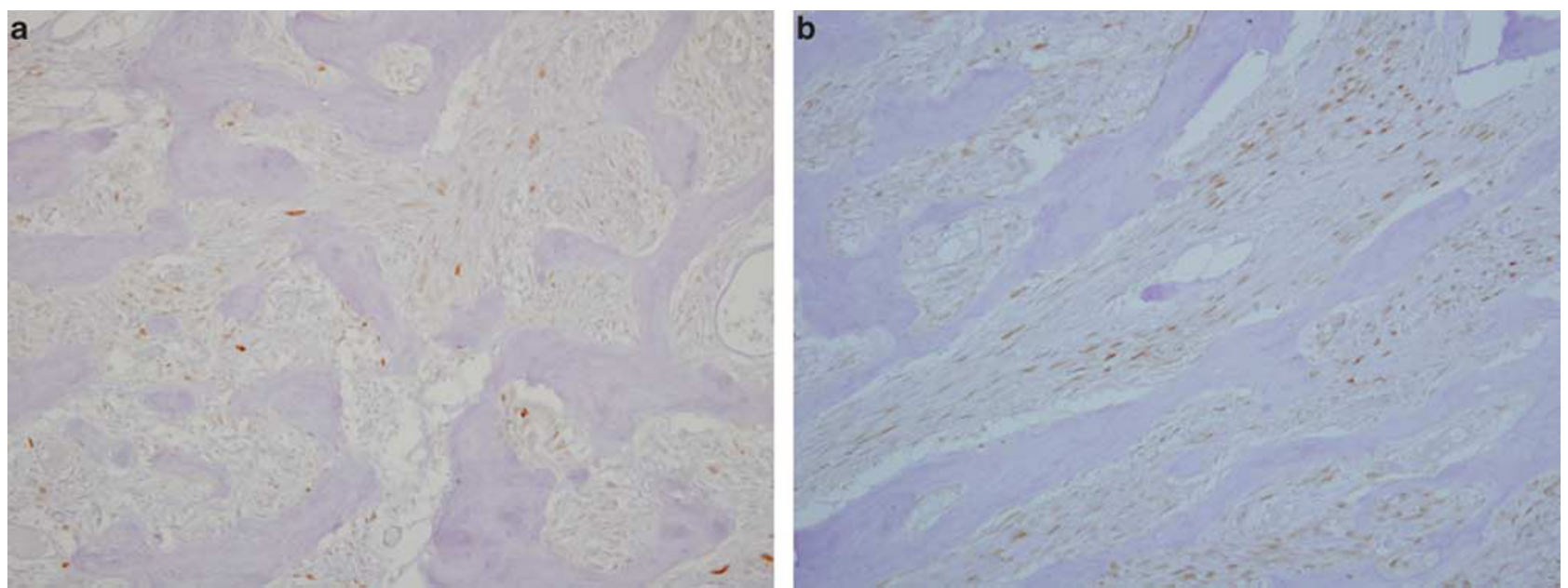

Figure 5 Murine double-minute type 2 (MDM2) and cyclin-dependent kinase 4 (CDK4) immunostaining in a case of low-grade osteosarcoma. The spindle cells are focally positive for MDM2 (a) and more diffusely stained with CDK4 (b) $(\times 200)$.
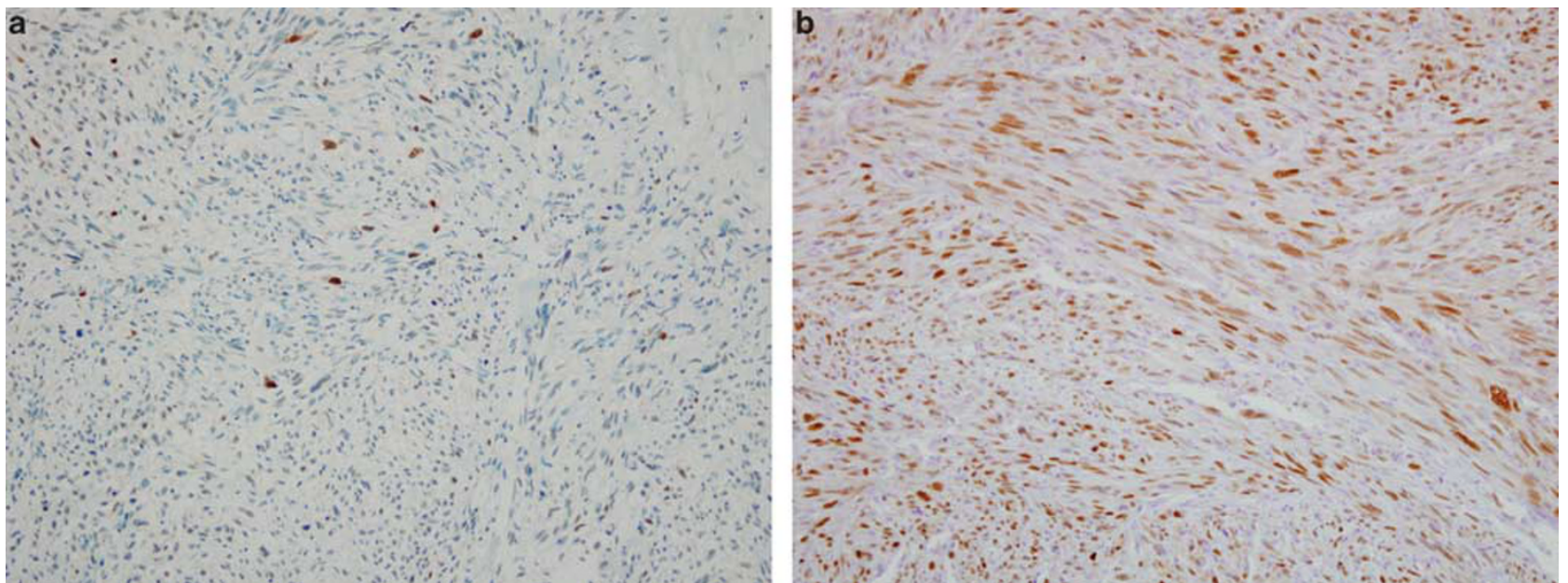

Figure 6 Murine double-minute type 2 (MDM2) and cyclin-dependent kinase 4 (CDK4) immunostaining in a case of dedifferentiated low-grade osteosarcoma (high-grade pleiomorphic spindle cell sarcomatous/malignant fibrous histiocytoma dedifferentiated areas). MDM2 staining (a) was more focal than CDK4 staining (b). The percentages of both MDM2- and CDK4-stained nuclei were higher than in well-differentiated areas $(\times 200)$. 


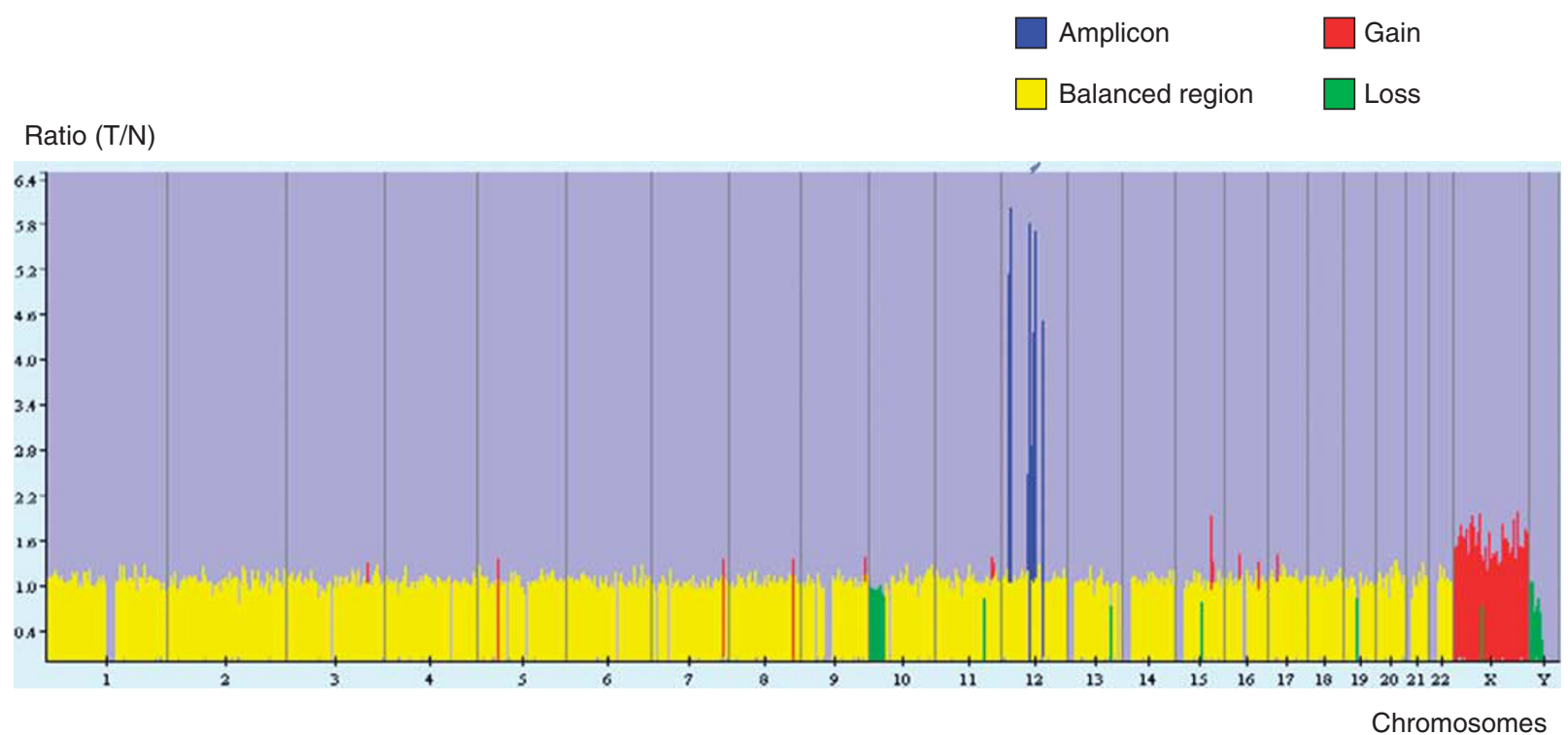

Figure 7 Comparative genomic hybridization array (aCGH) profile of case 42 showing chromosome 12q13-15 amplification containing cyclin-dependent kinase $4(C D K 4)$ and murine double-minute type 2 (MDM2) genes. Tumoral versus normal DNA ratio for each bacterial artificial chromosome (BAC) clone is plotted according to genome order (hg18 assembly). Balanced regions are represented in yellow, the regions gained are in red, the regions lost in green and amplicons in blue.

Table 5 CGH results of 18 low-grade osteosarcomas and comparison with MDM2 and CDK4 immunohistochemical status

\begin{tabular}{|c|c|c|c|c|c|}
\hline \multirow[t]{2}{*}{ Case no. } & \multirow[t]{2}{*}{ Histological diagnosis } & \multirow[t]{2}{*}{ Dedifferentiation } & \multirow{2}{*}{$\begin{array}{l}\text { CGH results- } \\
\text { amplification } \\
12 q 15\end{array}$} & \multicolumn{2}{|c|}{ Immunohistochemistry } \\
\hline & & & & $\begin{array}{c}\text { MDM2 } \\
\text { expression }\end{array}$ & $\begin{array}{c}\text { CDK4 } \\
\text { expression }\end{array}$ \\
\hline 28 & Low-grade central osteosarcoma & - & + & + & + \\
\hline 29 & Low-grade central osteosarcoma & - & + & + & + \\
\hline 30 & Parosteal osteosarcoma & + (osteosarcoma) & + & + & + \\
\hline 33 & Parosteal osteosarcoma & - & + & + & + \\
\hline 37 & Parosteal osteosarcoma & + (osteosarcoma) & + & - & + \\
\hline 38 & Parosteal osteosarcoma & - & + & - & + \\
\hline 42 & Parosteal osteosarcoma & - & + & - & - \\
\hline 43 & Parosteal osteosarcoma & - & + & - & - \\
\hline 44 & Parosteal osteosarcoma & - & + & + & + \\
\hline 45 & Parosteal osteosarcoma & - & + & + & - \\
\hline 49 & Low-grade central osteosarcoma & - & + & + & + \\
\hline 52 & Parosteal osteosarcoma & - & + & + & + \\
\hline 56 & Parosteal osteosarcoma & + (osteosarcoma) & + & + & + \\
\hline 57 & Low-grade central osteosarcoma & - & + & + & + \\
\hline 62 & Low-grade central osteosarcoma & - & + & + & + \\
\hline 66 & Parosteal osteosarcoma & - & + & + & + \\
\hline 68 & Parosteal osteosarcoma & + (osteosarcoma) & + & + & + \\
\hline 69 & Parosteal osteosarcoma & - & + & + & + \\
\hline
\end{tabular}

Abbreviations: CDK4, cyclin-dependent kinase 4; CGH, comparative genomic hybridization; MDM2, murine double-minute type 2.

were MDM2 and CDK4 immunohistochemistry staining negative (Table 5).

\section{FISH Analysis}

Of the benign fibro-osseous lesions analyzed $(n=15)$, only two cases were interpretable, and they did not show amplification of MDM2 consistent with the immunohistochemistry results. FISH results were interpretable in all cases of conventional high-grade osteosarcoma $(n=20)$, none of them showed amplification of $M D M 2$, and this was consistent with the immunohistochemistry results (Table 6).

\section{Q-PCR Analysis}

None of the fibrous or fibro-osseous lesions $(n=16)$ or the tumors of the control group tested $(n=3)$ showed amplification of MDM2, which was 
Table 6 FISH results and comparison with MDM2 and CDK4 immunohistochemical status

\begin{tabular}{|c|c|c|c|c|c|c|}
\hline \multirow[t]{2}{*}{ Histological diagnosis } & \multirow{2}{*}{$\begin{array}{l}\text { Total number } \\
\text { of tumors }\end{array}$} & \multicolumn{3}{|c|}{ FISH results-MDM2 amplification } & \multicolumn{2}{|c|}{ Immunohistochemistry } \\
\hline & & Amplification & $\begin{array}{c}\text { No } \\
\text { amplification }\end{array}$ & $\begin{array}{c}\text { Not } \\
\text { interpretable }\end{array}$ & $\begin{array}{l}\text { MDM2 } \\
\text { expression }\end{array}$ & $\begin{array}{c}\text { CDK4 } \\
\text { expression }\end{array}$ \\
\hline $\begin{array}{l}\text { Fibrous and fibro-osseous } \\
\text { bone lesions }\end{array}$ & 15 & 0 & 2 & 13 & 0 & 0 \\
\hline Conventional osteosarcomas & 20 & 0 & 0 & 0 & 0 & 0 \\
\hline
\end{tabular}

Abbreviations: CDK4, cyclin-dependent kinase 4; FISH, fluorescence in situ hybridization; MDM2, murine double-minute type 2.

Table 7 PCR results and comparison with MDM2 and CDK4 immunohistochemical status

\begin{tabular}{lccc}
\hline Histological diagnosis & $\begin{array}{c}\text { Total number of } \\
\text { tumors }\end{array}$ & $\begin{array}{c}\text { PCR results_MDM2 } \\
\text { amplification }\end{array}$ & \begin{tabular}{c} 
Immunohistochemistry \\
\cline { 2 - 3 }
\end{tabular} \\
expression & $\begin{array}{c}\text { CDK4 } \\
\text { expression }\end{array}$ \\
\hline $\begin{array}{l}\text { Fibrous and fibro-osseous bone lesions } \\
\text { High-grade primary bone sarcomas (two pleomorphic }\end{array}$ & 16 & 0 & 0 \\
$\begin{array}{l}\text { spindle cell sarcoma/malignant fibrous histiocytoma } \\
\text { and one leiomyosarcoma) }\end{array}$ & 3 & 0 & 0 \\
\hline
\end{tabular}

Abbreviations: CDK4, cyclin-dependent kinase 4; MDM2, murine double-minute type 2; PCR, polymerase chain reaction.

also consistent with the immunohistochemistry results (Table 7).

\section{Discussion}

Low-grade osteosarcomas are well-differentiated tumors, which are locally aggressive, but with limited potential for distant spread. However, the prognosis may be worsened by the occurrence of dedifferentiation that signifies progression towards a higher-grade osteosarcoma or heterologous sarcoma with metastatic potential. This dedifferentiation may occur either at presentation (synchronous type) or at the time of recurrence (metachronous type) without any difference in outcome between the two groups. ${ }^{7}$ In our study, 16/17 dedifferentiated low-grade osteosarcomas were synchronous tumors.

Low-grade central osteosarcoma and parosteal osteosarcoma share similar histological ${ }^{12}$ and ultrastructural features. ${ }^{22}$ They may arise simultaneously. ${ }^{23}$ Low-grade osteosarcomas are rare tumors characterized clinically by slow growth. They appear histologically as quiescent tumors with low cellularity. Atypia and mitoses are rare or absent. Cases of low-grade osteosarcoma are therefore often misdiagnosed as benign lesions. ${ }^{13,14}$ An experienced team can diagnose parosteal osteosarcoma on typical radiological features, that is, a heavily mineralized mass attached to the cortex of the distal posterior femur. In some cases, primary surgical resection without previous biopsy can then be proposed. Nevertheless, a significant number of parosteal osteosarcomas may develop at other locations and most low-grade central osteosarcomas have a misleading radiographic appearance at initial presentation. A low-grade osteosarcoma may both radiologically and histologically mimic a group of fibrous or fibro-osseous benign lesions involving the medullary canal or the bone surface. The main differential diagnoses for low-grade central osteosarcoma are, in practice, fibrous dysplasia ${ }^{3,6,11,12,24}$ and desmoplastic fibroma. ${ }^{3,12}$ The key factor differentiating low-grade central osteosarcoma from fibrous dysplasia is therefore the infiltrative growth pattern of the tumor, with permeation of the bone marrow and encasement of the pre-existing trabecular bone., ${ }^{3,4}$ However, this feature is rarely observed on a biopsy specimen. Usually any bone formation rules out desmoplastic fibroma and supports the diagnosis of low-grade central osteosarcoma. ${ }^{3}$ In everyday practice, differentiating both tumors can be difficult on a biopsy specimen: bone trabeculae can be sparse in some cases of low-grade osteosarcoma and reactive bone may sometimes be observed at the periphery of a desmoplastic fibroma. ${ }^{12}$ The infiltrative tumoral pattern, cortical disruption and soft-tissue extension cannot totally provide a differential diagnosis, but are more suggestive of the low-grade malignancy of a lowgrade central osteosarcoma. ${ }^{12,25}$ The presence of a cloud-like tumor matrix pattern on radiological findings, reflecting the presence of intra-tumoral bone formation, is also a good argument for lowgrade central osteosarcoma. Another pitfall when diagnosing low-grade central osteosarcoma is the liposclerosing myxofibrous tumor, a lesion possibly linked to fibrous dysplasia and characteristically 
located in the proximal femur, at the base of the femoral neck. ${ }^{26}$ It can also be (more rarely) confused with Paget's disease of the bone ${ }^{15}$ or with a giant bone island. The latter lesion is exclusively made of cortical haversian bone without any cell proliferation within the haversian canals. ${ }^{27}$

Several jawbone lesions may present a fibroosseous pattern with overlapping histological features, often making their diagnosis difficult. This group of lesions includes fibrous dysplasia such as those in the extra-gnathic skeleton and also more specific entities such as ossifying/cementifying fibroma and periapical cemental dysplasia. Low-grade osteosarcoma should be included in the differential diagnosis of these lesions. The osseous component of fibrous dysplasia of the jawbone may show a different pattern, with long and anastomosing bone trabeculae mimicking the tumor osteogenesis of low-grade osteosarcoma. Moreover, atypical microscopic features have been described in some cases of fibro-osseous lesions of the jawbone. ${ }^{28}$

Parosteal osteosarcoma should be differentiated from osteochondromas, benign paraosteal and periosteal bone lesions and periosteal processes, and from reactive soft-tissue processes adhering secondarily to the bone surface. These lesions include Nora's lesions, fracture callus, myositis ossificans, soft-tissue fibromatosis, reactive periostitis and ossifying fibromyxoid tumor. ${ }^{9,29-31}$ The cartilaginous component of parosteal osteosarcoma may be significant and, in approximately $25 \%$ of cases, is located on the surface of the tumor as a cartilaginous cap mimicking osteochondroma. ${ }^{2}$ Lin et $a l^{31}$ reported six cases of osteochondroma-like parosteal osteosarcoma initially misdiagnosed as benign lesions on pathology evaluation. Parosteal osteosarcoma can be confused with Nora's lesions mainly in cases involving the long bones or in cases with unusual radiographic features. ${ }^{29,32}$ In later phases of reactive soft-tissue and periosteal processes such as myositis ossificans or reactive periostitis, bone trabeculae appear more mature and these lesions may be confused with low-grade osteosarcoma on a biopsy sample from which the zonal architecture may be missing. ${ }^{4}$ Rare cases of paraosteal osteomas involving long bones may also simulate parosteal osteosarcoma. However, they histologically consist of dense sclerotic lamellar bone with haversian systems, without any spindle cell proliferation. ${ }^{33}$ Finally, protuberans fibrous dysplasia is a rare variant of fibrous dysplasia mimicking surface lesions of the bone. It is a benign fibro-osseous exophytic mass with heterogeneous sclerosis located eccentrically in the intra-medullary cavity of an adjacent bone. ${ }^{34}$ Without detailed radiographic imaging and meticulous histological examination, this benign lesion is likely to be misdiagnosed, especially in parosteal osteosarcoma.

Significant sampling of low-grade osteosarcoma is very important because it may be difficult or impossible to distinguish it histologically from such benign bone lesions on limited samples from a core biopsy or even more so from needle aspiration. ${ }^{12}$ An open surgical biopsy is often necessary to obtain a large tumor sample, but even this may be sometimes insufficient. Muramatsu et $a l^{24}$ reported a case for which the initial microscopic diagnosis was fibrous dysplasia on open biopsy, and Bertoni et $a l^{12}$ reported a case for which the original histological diagnosis was Paget's disease and/or fibrous dysplasia. The diagnosis of low-grade osteosarcoma was made a few years later in the recurrence in the bone and soft tissue and at the time of amputation with areas of high-grade osteosarcoma (dedifferentiation). Low-grade osteosarcoma characterized by a deceptively benign-looking histological appearance is an important diagnostic pitfall for pathologists that may often lead to underdiagnosis of malignancy. Finding new, easily performed diagnostic markers is thus a challenge for management of low-grade osteosarcoma.

Several markers (ie, actin, osteonectin, osteocalcin, c-fos and c-jun oncoproteins and ezrin) were shown to be of no value in distinguishing lowgrade osteosarcoma from benign fibro-osseous lesions. ${ }^{18,35-40}$ Okada et $a l^{41}$ studied eight cases of low-grade central osteosarcomas retrospectively and showed that proliferative cell activity evaluated by AgNOR and MIB-1 immunohistochemical staining was significantly higher in cases of low-grade central osteosarcoma than in fibrous dysplasia and might be helpful in differentiating low-grade central osteosarcoma from fibrous dysplasia. Pollandt et $a l^{42}$ showed that Gsalpha gene mutations were a constant finding in cases of monostotic fibrous dysplasia. Nevertheless, they also reported a Gsalpha gene mutation in one of five cases of fibrous dysplasialike low-grade central osteosarcoma studied. Such mutations appear to be not totally specific of fibrous dysplasia.

In contrast to the complex karyotypes previously reported in conventional osteosarcoma, ${ }^{43}$ low-grade osteosarcoma is characterized by a simple karyotype with supernumerary ring chromosomes ${ }^{19,44-46}$ and over-representation of $12 \mathrm{q}$ sequences on CGH. ${ }^{16,17,21}$ Ring chromosomes are composed of 12q13-15 clusters containing MDM2 and CDK4 and are due to telomeric deletions. ${ }^{16,47}$ Similar cytogenetic abnormalities have previously been described in other low-grade malignancy mesenchymal lesions such as well-differentiated liposarcoma. ${ }^{48}$ Overexpression and gene amplification of MDM2 and CDK4 located on chromosome 12q13-15 have been reported to occur in various human sarcomas as the pathway of tumorigenesis or tumor progression. ${ }^{49,50}$ As a result, two major growth regulation pathways may be inhibited. MDM2 may downregulate the p53mediated growth control and CDK4 may affect retinoblastoma tumor suppressor protein (pRb)mediated events. ${ }^{51}$ These genes have been reported to be related to amplification of the 12q13-15 region, but they are located in two discontinuous regions, 
which are closely linked and frequently co-amplified. ${ }^{48}$ Amplification of $C D K 4$ and MDM2 can lead to deregulation of the cell cycle and may be an important step in tumor progression. Although some studies have reported $12 \mathrm{q}$ amplification in conventional high-grade osteosarcomas, with a frequency ranging from 0 to $27 \%,{ }^{19,50,52-59}$ the majority have indicated that amplification of MDM2 and CDK4 is a typical feature of parosteal osteosarcoma and lowgrade central osteosarcoma. ${ }^{16,17,19,21,47,52,60,61}$ One study showed a high level of CDK4 and MDM2 amplification in a series of nine osteosarcomas of the jaw, including five intermediate and high-grade osteosarcomas. ${ }^{51}$ However, gnathic osteosarcomas have particular clinical and biological profiles that differ from conventional high-grade osteosarcomas of long bones and that require further specific investigation. In our series, we did not study lowgrade osteosarcomas of the jaw. However, the negativity of CDK4 and MDM2 immunostaining of 10 fibrous and fibro-osseous maxillary lesions shows that MDM2 and CDK4 can help to differentiate these benign lesions from osteosarcomas. Low-grade osteosarcoma studies analyzing MDM2 expression by immunohistochemistry have reported a frequency range of $22-70$ and $66-92 \%$ for CDK4. ${ }^{20,21,59-61}$ This wide range can be explained by technical differences, such as formalin fixation, decalcification of tissues, antibody dilution and antigen retrieval method. The biomolecular study of MDM2 amplification in low-grade osteosarcomas, including aCGH, FISH and PCR, has revealed a frequency range of $19-100$ and $12-80 \%$ for CDK $4 .{ }^{19,47,52,59,60}$ The highest amplification frequencies were obtained with Q-PCR ${ }^{52}$ on frozen samples and with FISH and Southern blotting ${ }^{19,47}$ on unspecified material. DNA extraction from paraffin blocks can explain the disappointing results with Q-PCR in two studies. ${ }^{59,60}$

In our study, overexpression of MDM2 and/or CDK4 was observed by immunohistochemistry in $89 \%$ of cases of low-grade osteosarcoma (sensitivity) with a specificity of $100 \%$, with or without dedifferentiation. All low-grade central osteosarcomas showed expression of MDM2 and/or CDK4. The results obtained from our eight patients are promising and will need further investigation. These results were consistent with MDM2 amplification findings provided by aCGH-tested low-grade osteosarcomas $(n=18)$. Neither the fibrous/fibro-osseous lesions tested by Q-PCR $(n=16)$ or FISH $(n=15)$ nor the tumors of the control group tested by FISH or QPCR showed MDM2 amplification. Moreover, investigation of $M D M 2$ amplification by aCGH provided a correct diagnosis in two cases of low-grade osteosarcoma whose immunohistochemical study was negative (Table 3). Consequently, although molecular study often needs to be executed on frozen samples and is not easily available in a routine setting, this technique is required in certain cases to improve overall diagnostic sensitivity.
As in our study, a recent analysis tested MDM2 and CDK4 immunostaining in low-grade osteosarcomas (23 cases) and in benign fibro-osseous lesions (40 cases). ${ }^{61}$ Their results confirmed the very good sensitivity of the test $(100 \%)$ and its specificity $(97.5 \%)$. However, the number of cases studied was lower and the MDM2 and CDK4 amplification status was not investigated. This would have been particularly interesting in the immunostaining-positive Nora's lesion in this study.

Our results confirm that 12q13-15 genes are involved in tumor progression in low-grade osteosarcoma. Concurrent amplification and overexpression of these genes might help to diagnose low-grade osteosarcomas, particularly when they mimic benign fibro-osseous lesions. In the 10 cases of low-grade osteosarcoma with a histologically unusual growth pattern simulating benign fibro-osseous lesions, nine were positive on MDM2 and/or CDK4 immunostaining. The case of MDM2-CDK4-negative immunostaining (fibrous dysplasia-like low-grade osteosarcoma) showed MDM2 amplification on CGH analysis.

Dedifferentiated areas in dedifferentiated lowgrade osteosarcomas and other primary high-grade bone sarcomas such as conventional high-grade osteosarcomas can share histological features even if they have a different oncogenesis. In our study, amplification/overexpression of MDM2 and CDK4 was clearly observed in differentiated and dedifferentiated low-grade osteosarcoma components, in contrast to the control group of high-grade conventional osteosarcomas $(n=20)$ and other rare subtypes of intra-osseous primary sarcomas $(n=3)$. Although Wold et $a l^{10}$ showed that the prognosis for patients with dedifferentiated parosteal osteosarcoma appears to be similar to that of patients with high-grade centromedullary osteosarcoma, some recent studies have suggested that the prognosis of dedifferentiated low-grade osteosarcomas may be better than that of primary conventional centromedullary or surface high-grade osteosarcomas. ${ }^{5,7}$ This potential difference in malignant behavior may be explained by the different changes in oncogenesis in these two kinds of tumor. In summary, proving overexpression of MDM2 and CDK4 in dedifferentiated low-grade osteosarcomas may allow pathologists to distinguish dedifferentiated low-grade osteosarcomas from conventional high-grade osteosarcomas, although the clinical outcome remains unclear. ${ }^{7}$ However, the difference would be important with the discovery of targeted molecular therapy. ${ }^{62}$

Despite standardized protocols, the formalin fixation of tissues and decalcification by acid-based products may result in the failure of FISH by hydrolysis of DNA and in the loss of sensitivity of immunohistochemical analysis. For example, of six cases of low-grade osteosarcoma with both biopsy and resection material available (data not shown), the IHC results were similar for both specimens in 
our series, except for one case. Positive tumor cell immunostaining was observed for both MDM2 and CDK4 on biopsy sample, thus confirming the diagnosis. However, this immunohistochemical positivity was not found in the surgical resection specimen, whereas there was MDM2 amplification according to molecular studies on both specimens. This can be explained by the longer decalcification period required for the surgical specimen. Moreover, the failure of FISH for 13/15 fibro-osseous lesions analyzed can also be explained by the acid decalcification. Using chelating agents such as EDTA for decalcification might circumvent this problem. Decalcification in EDTA has little or no effect on tissues other than on the bone mineral matrix itself. Nevertheless, the application of EDTA as a decalcifying agent in a routine setting is hampered by the long period required for incubation. ${ }^{63}$ New methods such as decalcification in EDTA using a microwave oven and ultrasonic decalcification ${ }^{64}$ are reported to considerably reduce the time of decalcification and preserve antigenic sites, DNA and mRNA. ${ }^{63}$

In conclusion, overexpression and amplification of MDM2 and CDK4 are confirmed in low-grade osteosarcomas (parosteal osteosarcomas, low-grade central osteosarcomas and dedifferentiated lowgrade osteosarcomas). The evaluation of MDM2 and CDK4 overexpression by immunohistochemistry appears to be a valuable diagnostic tool to distinguish low-grade osteosarcomas from lookalike benign fibrous and fibro-osseous lesions with quite good sensitivity. These markers are particularly interesting when there are limited samples from a core biopsy or when the initial presentation is unrecognized. MDM2 and CDK4 are also markers distinguishing between dedifferentiated low-grade osteosarcomas and conventional high-grade osteosarcomas. Improving the sensitivity of these markers by the use of chelating agents such as EDTA should be tested in further studies. In some cases, the use of molecular analysis such as CGH or Q-PCR on frozen samples is necessary to detect MDM2 and/or $C D K 4$ gene amplification.

\section{Disclosure/conflict of interest}

The authors declare no conflict of interest.

\section{References}

1 Fletcher C, Unni KK, Mertens F. World Health Organisation Classification Pathology and Genetics. Tumours of the Soft Tissue and Bone. IARC Press: Lyon, France, 2004.

2 Okada K, Frassica FJ, Sim FH, et al. Parosteal osteosarcoma. A clinicopathological study. J Bone Joint Surg Am 1994;76:366-378.

3 Kurt AM, Unni KK, McLeod RA, et al. Low-grade intraosseous osteosarcoma. Cancer 1990;65:1418-1428.
4 Antonescu CR, Huvos AG. Low-grade osteogenic sarcoma arising in medullary and surface osseous locations. Am J Clin Pathol 2000;114(Suppl):S90-103.

5 Schwab JH, Antonescu CR, Athanasian EA, et al. A comparison of intramedullary and juxtacortical low-grade osteogenic sarcoma. Clin Orthop Relat Res 2008;466:1318-1322.

6 Choong PF, Pritchard DJ, Rock MG, et al. Low grade central osteogenic sarcoma. A long-term followup of 20 patients. Clin Orthop Relat Res 1996;322:198-206.

7 Bertoni F, Bacchini P, Staals EL, et al. Dedifferentiated parosteal osteosarcoma: the experience of the Rizzoli Institute. Cancer 2005;103:2373-2382.

8 Campanacci M, Picci P, Gherlinzoni F, et al. Parosteal osteosarcoma. 1. BoneJoint Surg 1984;66:313-321.

9 Ritschl P, Wurnig C, Lechner G, et al. Parosteal osteosarcoma. 2-23-year follow-up of 33 patients. Acta Orthop Scand 1991;62:195-200.

10 Wold LE, Unni KK, Beabout JW, et al. Dedifferentiated parosteal osteosarcoma. J Bone Joint Surg Am 1984; 66:53-59.

11 Ogose A, Hotta T, Emura I, et al. Repeated dedifferentiation of low-grade intraosseous osteosarcoma. Hum Pathol 2000;31:615-618.

12 Bertoni F, Bacchini P, Fabbri N, et al. Osteosarcoma. Low-grade intraosseous-type osteosarcoma, histologically resembling parosteal osteosarcoma, fibrous dysplasia, and desmoplastic fibroma. Cancer 1993;71: 338-345.

13 Grimer RJ, Carter SR, Spooner D, et al. Diagnosing musculoskeletal tumours. Sarcoma 2001;5:89-94.

14 Mankin HJ, Lange TA, Spanier SS. The hazards of biopsy in patients with malignant primary bone and soft tissue tumours. J Bone Joint Surg 1982;64: 1121-1127.

15 Franchi A, Bacchini P, Della Rocca C, et al. Central low-grade osteosarcoma with pagetoid bone formation: a potential diagnostic pitfall. Mod Pathol 2004;17: 288-291.

16 Szymanska J, Mandahl N, Mertens F, et al. Ring chromosomes in parosteal osteosarcoma contain sequences from 12q13-15: a combined cytogenetic and comparative genomic hybridization study. Genes Chromosomes Cancer 1996;16:31-34.

17 Tarkkanen M, Böhling T, Gamberi G, et al. Comparative genomic hybridization of low-grade central osteosarcoma. Mod Pathol 1998;11:421-426.

18 Franchi A, Calzolari A, Zampi G. Immunohistochemical detection of c-fos and c-jun expression in osseous and cartilaginous tumours of the skeleton. Virchows Arch 1998;432:515-519.

19 Gisselsson D, Palsson E, Hoglund M, et al. Differentially amplified chromosome 12 sequences in low- and high-grade osteosarcoma. Genes Chromosomes Cancer 2002;33:133-140.

20 Ragazzini P, Gamberi G, Benassi MS, et al. Analysis of SAS gene and CDK4 and MDM2 proteins in low-grade osteosarcoma. Cancer Detect Prev 1999;23:129-136.

21 Tarkkanen M, Karhu R, Kallioniemi A, et al. Gains and losses of DNA sequences in osteosarcomas by comparative genomic hybridization. Cancer Res 1995;55:1334-1338.

22 Steiner GC, Forest M, Vacher-Lavenu MC. Ultrastructure of low-grade intraosseous osteosarcoma of bone: a comparative study with fibrous dysplasia and parosteal osteosarcoma. Ultrastruct Pathol 2006;30: 293-299. 
23 Arkader A, Huvos AG, Healey JH. Multifocal synchronous low-grade intramedullary and juxtacortical osteogenic sarcoma. Clin Orthop Relat Res 2007;465 :265-270.

24 Muramatsu K, Hashimoto T, Seto S, et al. Low-grade central osteosarcoma mimicking fibrous dysplasia: a report of two cases. Arch Orthop Trauma Surg 2008;128:11-15.

25 Andresen KJ, Sundaram M, Unni KK, et al. Imaging features of low-grade central osteosarcoma of the long bones and pelvis. Skeletal Radiol 2004;33:373-379.

26 Heim-Hall JM, Williams RP. Liposclerosing myxofibrous tumour: a traumatized variant of fibrous dysplasia? Report of four cases and review of the literature. Histopathology 2004;45:369-376.

27 Park HS, Kim JR, Lee SY, et al. Symptomatic giant $(10-\mathrm{cm})$ bone island of the tibia. Skeletal Radiol 2005;34:347-350.

28 Koury ME, Regezi JA, Perrott DH, et al. 'Atypical' fibroosseous lesions: diagnostic challenges and treatment concepts. Int J Oral Maxillofac Surg 1995;24: 162-169.

29 Subasi M, Kapukaya A, Buyukbayram H, et al. Unusual benign bone lesion simulating parosteal osteosarcoma. J Orthop Sci 2006;11:529-532.

30 Ogose A, Otsuka H, Morita T, et al. Ossifying fibromyxoid tumor resembling parosteal osteosarcoma. Skeletal Radiol 1998;27:578-580.

31 Lin J, Yao L, Mirra JM, et al. Osteochondroma-like parosteal osteosarcoma: a report of six cases of a new entity. Am J Roentgenol 1998;170:1571-1577.

32 Soubeyrand M, de Pinieux G, Biau D, et al. Bizarre parosteal osteochondromatous proliferation (Nora's lesion): two cases. Rev Chir Orthop Reparatrice Appar Mot 2007;93:494-500.

33 Chikuda H, Goto T, Ishida T, et al. Juxtacortical osteoma of the ulna. J Orthop Sci 2002;7:721-723.

34 Yamaguchi T, Shimizu K, Koguchi Y, et al. Low-grade central osteosarcoma of the rib. Skeletal Radiol 2005; 34:490-493.

35 Franchi A, Comin CE, Santucci M. Submicroscopic and immunohistochemical profile of surface osteosarcomas. Ultrastruct Pathol 1999;23:233-240.

36 Serra M, Morini MC, Scotlandi K, et al. Evaluation of osteonectin as a diagnostic marker of osteogenic bone tumors. Hum Pathol 1992;23:1326-1331.

37 Sakamoto A, Oda Y, Iwamoto Y, et al. A comparative study of fibrous dysplasia and osteofibrous dysplasia with regard to expressions of c-fos and c-jun products and bone matrix proteins: a clinicopathologic review and immunohistochemical study of c-fos, c-jun, type I collagen, osteonectin, osteopontin, and osteocalcin. Hum Pathol 1999;30:1418-1426.

38 Toyosawa S, Yuki M, Kishino M, et al. Ossifying fibroma vs fibrous dysplasia of the jaw: molecular and immunological characterization. Mod Pathol 2007; 20:389-396.

39 Vermeulen AH, Vermeer C, Bosman FT. Histochemical detection of osteocalcin in normal and pathological human bone. J Histochem Cytochem 1989;37: 1503-1508.

40 Park HR, Jung WW, Bacchini P, et al. Ezrin in osteosarcoma: comparison between conventional high-grade and central low-grade osteosarcoma. Pathol Res Pract 2006;202:509-515.

41 Okada K, Nishida J, Morita T, et al. Low-grade intraosseous osteosarcoma in northern Japan: advan- tage of AgNOR and MIB-1 staining in differential diagnosis. Hum Pathol 2000;31:633-639.

42 Pollandt K, Engels C, Kaiser E, et al. Gsalpha gene mutations in monostotic fibrous dysplasia of bone and fibrous dysplasia-like low-grade central osteosarcoma. Virchows Arch 2001;439:170-175.

43 Bridge JA, Nelson M, McComb E, et al. Cytogenetic findings in 73 osteosarcoma specimens and a review of the literature. Cancer Genet Cytogenet 1997;95:74-87.

44 Sinovic JF, Bridge JA, Neff JR. Ring chromosome in parosteal osteosarcoma. Clinical and diagnostic significance. Cancer Genet Cytogenet 1992;62:50-52.

45 Mertens F, Mandahl N, Orndal C, et al. Cytogenetic findings in 33 osteosarcomas. Int J Cancer 1993;55: 44-50.

46 Werner M, Rieck J, Delling G. Cytogenetic changes in low grade central osteosarcomas. Verh Dtsch Ges Pathol 1998;82:189-194.

47 Wunder JS, Eppert K, Burrow SR, et al. Co-amplification and overexpression of CDK4, SAS and MDM2 occurs frequently in human parosteal osteosarcomas. Oncogene 1999;18:783-788.

48 Pedeutour F, Maire G, Sirvent N. From cytogenetics to cytogenomics of adipose tissue tumors: Malignant adipose tissue tumor. Bull Cancer 2004;91:317-323.

49 Khatib ZA, Matsushime $\mathrm{H}$, Valentine $\mathrm{M}$, et al. Coamplification of the CDK4 gene with MDM2 and GLI in human sarcomas. Cancer Res 1993;53: 5535-5541.

50 Ladanyi $\mathrm{M}$, Cha $\mathrm{C}$, Lewis $\mathrm{R}$, et al. MDM2 gene amplification in metastatic osteosarcoma. Cancer Res 1993;53:16-18.

51 Lopes MA, Nikitakis NG, Ord RA, et al. Amplification and protein expression of chromosome 12q13-15 genes in osteosarcomas of the jaws. Oral Oncol 2001;37:566-571.

52 Mejia-Guerrero S, Quejada M, Gokgoz N, et al. Characterization of the $12 \mathrm{q} 15$ MDM2 and 12q13-14 CDK4 amplicons and clinical correlations in osteosarcoma. Genes Chromosomes Cancer 2010;49:518-525.

53 Lonardo F, Ueda T, Huvos AG, et al. p53 and MDM2 alterations in osteosarcomas: correlation with clinicopathologic features and proliferative rate. Cancer 1997; 79:1541-1547.

54 Oliner JD, Kinzler KW, Meltzer PS, et al. Amplification of a gene encoding a p53-associated protein in human sarcomas. Nature 1992;358:80-83.

55 Tsuchiya T, Sekine K, Hinohara S, et al. Analysis of the p16INK4, p41ARF, p15, TP53, and MDM2 genes and their prognostic implications in osteosarcoma and Ewing sarcoma. Cancer Genet Cytogenet 2000;120:91-98.

56 Yokoyama R, Schneider-Stock R, Radig $\mathrm{K}$, et al. Clinicopathologic implications of MDM2, p53 and K-ras gene alterations in osteosarcomas: MDM2 amplification and p53 mutations found in progressive tumors. Pathol Res Pract 1998;194:615-621.

57 Kawaguchi K, Oda Y, Sakamoto A, et al. Molecular analysis of p53, MDM2, and H-ras genes in osteosarcoma and malignant fibrous histiocytoma of bone in patients older than 40 years. Mod Pathol 2002;15: 878-888.

58 Momand J, Jung D, Wilczynski S, et al. The MDM2 gene amplification database. Nucleic Acids Res 1998;26:3453-3459.

59 Park HR, Jung WW, Bertoni F, et al. Molecular analysis of p53, MDM2 and H-ras genes in low-grade central osteosarcoma. Pathol Res Pract 2004;200:439-445. 
60 Gamberi G, Ragazzini P, Benassi MS, et al. Analysis of 12q13-15 genes in parosteal osteosarcoma. Clin Orthop Relat Res 2000;377:195-204.

61 Yoshida A, Ushiku T, Motoi T, et al. Immunohistochemical analysis of MDM2 and CDK4 distinguishes low-grade osteosarcoma from benign mimics. Mod Pathol 2010;23:1279-1288.

62 Zhang, Wang H. MDM2 oncogene as a novel target for human cancer therapy. Curr Pharm Des 2000;6:393-416.
63 Alers JC, Krijtenberg P-J, Vissers KJ, et al. Effect of bone decalcification procedures on DNA in situ hybridization and comparative genomic hybridization: EDTA is highly preferable to a routinely used acid decalcifier. J Histochem Cytochem 1999;47:703-709.

64 Reineke T, Jenni B, Abdou MT, et al. Ultrasonic decalcification offers new perspectives for rapid FISH, DNA, and RT-PCR analysis in bone marrow trephines. Am J Surg Pathol 2006;30:892-896. 